\title{
Some Remarks on the Self-Exponential Function: Minimum Value, Inverse Function, and Indefinite Integral
}

\author{
J. L. González-Santander and G. Martín \\ Departamento de Ciencias Experimentales y Matemáticas, Universidad Católica de Valencia "San Vicente Mártir", \\ C/Guillem de Castro 96, 46001 Valencia, Spain
}

Correspondence should be addressed to J. L. González-Santander; martinez.gonzalez@ucv.es

Received 9 September 2014; Accepted 10 November 2014; Published 23 November 2014

Academic Editor: Shamsul Qamar

Copyright (C) 2014 J. L. González-Santander and G. Martín. This is an open access article distributed under the Creative Commons Attribution License, which permits unrestricted use, distribution, and reproduction in any medium, provided the original work is properly cited.

Considering the function $x^{x}$ as a real function of real variable, what is its minimum value? Surprisingly, the minimum value is reached for a negative value of $x$. Furthermore, considering the function $f_{\beta}(x)=x^{-\beta x}, \beta \in \mathbb{R}$ and $x>0$, two different expressions in closed form for the inverse function $f_{\beta}^{-1}$ can be obtained. Also, two different series expansions for the indefinite integral of $f_{\beta}$ and $f_{\beta}^{-1}$ are derived. The latter does not seem to be found in the literature.

\section{Introduction}

Let us consider the following real function of real variable, $f: \mathbb{R} \rightarrow \mathbb{R}:$

$$
y=f(x)=x^{x}=\exp (x \log x),
$$

and let us pose the following questions.

(1) What is the minimum value of $f(x)$ ?

(2) Can its inverse function be expressed in closed form?

(3) Is its indefinite integral known?

The function $f(x)$ is termed as self-exponential function in [1, Section 26:14] and coupled exponential function in [2, Equation 01.20], using in the latter the notation $f(x)=$ $\operatorname{cxt}(x)$. Probably, the most well-known property of $f(x)$ is just its great growth rate. In fact, the rate of increase of $f(x)$ as $x \rightarrow \infty$ is greater than the exponential function or the factorial function [3, Chapter I. section 5]

$$
x^{x}>x !>e^{x} .
$$

Regarding the domain of $f(x)$, in [1, Section 26:14], $f(x)$ is only defined as a real function for positive values of $x$, and [2, p. 10] states that, for arguments less than zero, $f(x)$ is complex except for negative integers. However, [4] says that, for $x<0, f(x)$ is only defined if $x$ can be written as $-p / q$, where $p$ and $q$ are positive integers and $q$ is odd. We will use this fact later on in order to answer the first question.

This paper is organized such that each section is devoted to each of the questions raised above.

\section{Minimum Value}

The usual way to answer the first question is just to solve the equation $f^{\prime}(x)=0$; that is,

$$
\exp (x \log x)(\log x+1)=0,
$$

so

$$
x=\frac{1}{e} .
$$

Since

$$
f^{\prime \prime}\left(\frac{1}{e}\right)=e^{1-e^{-1}}>0,
$$

then (4) is a local minimum. Moreover, since there are no more local extrema and $f$ is a smooth function, (4) is the global minimum; thus,

$$
\min f(x)=f\left(\frac{1}{e}\right)=e^{-1 / e} \approx 0.692201 .
$$


Nonetheless, this reasoning fails, because it does not take into account negative values of $x$. Therefore, we need to define first the domain of $f(x)$ for negative values of $x$. Despite the fact that this is essentially done in [4], in order to answer the first question, we provide the following derivation.

2.1. Domain of $f(x)$. Let us consider first the case $x=0$, where the function $f(x)$ does not exist. However, applying L'Hôpital's rule, the following limit is finite:

$$
\lim _{x \rightarrow 0^{+}} x^{x}=1
$$

Nevertheless, the right derivative of $f(x)$ at $x=0$ is infinite:

$$
\lim _{x \rightarrow 0^{+}} f^{\prime}(x)=\lim _{x \rightarrow 0^{+}} x^{x}(\log x+1)=-\infty
$$

For $x \neq 0$, we may rewrite $f(x)$ by using the signum function $\operatorname{sgn}(x)$ as

$$
\begin{aligned}
f(x) & =\exp (x \log (\operatorname{sgn}(x)|x|)) \\
& =|x|^{x} \exp (x \log (\operatorname{sgn} x)) .
\end{aligned}
$$

Now, for $x \neq 0$ and $n \in \mathbb{Z}$, we have

$$
\begin{aligned}
\log (\operatorname{sgn} x) & = \begin{cases}0, & x>0 \\
i \pi+i 2 \pi(n-1), & x<0\end{cases} \\
& =i \pi(2 n-1) \theta(-x),
\end{aligned}
$$

where $\theta(x)$ is the Heaviside function. Therefore, by applying Euler's formula $e^{i \theta}=\cos \theta+i \sin \theta$, we obtain

$$
\begin{aligned}
& f(x) \\
& =|x|^{x} \exp (i \pi(2 n-1) \theta(-x) x) \\
& = \begin{cases}|x|^{x}, & x>0 \\
|x|^{x}[\cos (\pi(2 n-1) x)+i \sin (\pi(2 n-1) x)], & x<0 .\end{cases}
\end{aligned}
$$

Since $f(x)$ is a real function, the complex part of (11) has to be zero. For $x>0, f(x)$ is never complex, and for $x<0$ the complex part of $f(x)$ is zero when

$$
\sin (\pi(2 n-1) x)=0 \longrightarrow x=-\frac{m}{2 n-1}<0, \quad n, m \in \mathbb{Z}^{+} .
$$

Therefore, substituting (12) into (11), the function $f(x)$ is given by

$$
f(x)= \begin{cases}x^{x}, & x>0 \\ (-1)^{m}|x|^{x}, & x=-\frac{m}{2 n-1}<0, \quad n, m \in \mathbb{Z}^{+}\end{cases}
$$

and its domain is

$$
\operatorname{Dom} f=\mathbb{R}^{+} \cup\left\{x=-\frac{m}{2 n-1}, n, m \in \mathbb{Z}^{+}\right\} .
$$

Notice that, despite the fact we have considered the $\log (x)$ function as a multivalued function in (10), $f(x)$ is a singlevalued function in (13), because we are considering $f(x)$ as a real function. Figure 1 shows the plot of $f(x)$. According to (13), for $x<0$, the plot of $f(x)$ lies on the following curves:

$$
g_{ \pm}(x)= \pm|x|^{x}= \pm \exp (x \log (-x)) \text {, }
$$

with a numerable infinite number of points. Notice that + and - signs in (15) occur for $m$ even and odd positive integers in (13), respectively.

2.2. Minimum Value of $f(x)$. In order to calculate the minimum value of $f(x)$, for $x<0$, let us solve the equation $g_{ \pm}^{\prime}(x)=0$; that is,

$$
\pm \exp (x \log (-x))[\log (-x)+1]=0 .
$$

Thus,

$$
x=-\frac{1}{e} .
$$

Notice that

$$
g_{ \pm}^{\prime \prime}\left(\frac{-1}{e}\right)=\mp e^{1+e^{-1}}
$$

So, $g_{+}(x)$ has a maximum and $g_{-}(x)$ has a minimum in (17), which agrees with Figure 1 . However, $-1 / e \notin \operatorname{Dom} f$, so we have to get the best rational approximation $-p / q$ to $-1 / e$ in such a way that $-p / q \in \operatorname{Dom} f$. Moreover, since the minimum lies on the $g_{-}(x)$ curve, $p$ and $q$ must be both odd positive integers. In order to do so, let us consider the sequence

$$
\begin{array}{r}
a_{k}\left(x_{0}\right)=-\frac{\left\lfloor 10^{k}\left|x_{0}\right|\right\rfloor+\left\lfloor 10^{k}\left|x_{0}\right|\right\rfloor(\bmod 2)+1}{10^{k}-1}, \\
x_{0} \in \mathbb{R}, \quad k \in \mathbb{N},
\end{array}
$$

where $\lfloor x\rfloor$ denotes the floor function. Notice that the numerator and the denominator of (19) are both odd, so $a_{k}(x)=$ $-p / q$ irreducible, with $p, q$ being odd positive integers. Therefore, $a_{k}\left(x_{0}\right)$ is a sequence of rational numbers for which $f\left(a_{k}\left(x_{0}\right)\right)$ lies on the curve $g_{-}(x)$. Also, $a_{k}\left(x_{0}\right)$ is a monotonic decreasing sequence that satisfies

$$
\lim _{k \rightarrow \infty} a_{k}\left(x_{0}\right)=-\left|x_{0}\right|
$$

Defining now the rational number, $r \in \mathbb{Q}$,

$$
r=\min _{k \in \mathbb{N}}\left\{a_{k}\left(\frac{-1}{e}\right)\right\} .
$$

Then, the minimum value of $f(x)$ is

$$
\min _{x \in \operatorname{Dom} f} f(x)=f(r) \approx-1.44467,
$$

which is different from (6), as aforementioned. 


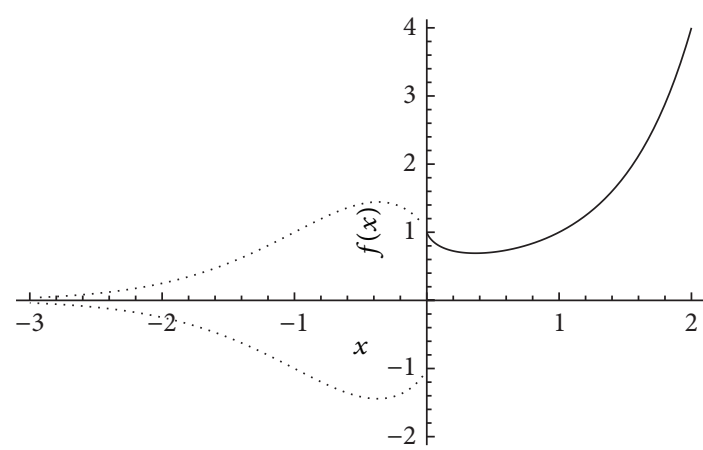

FIgURE 1: Plot of $x^{x}$ as a real function of real variable.

\section{Inverse Function}

About the second question, a closed form expression for the inverse function does not seem to be found in the usual literature (see [2, Chapter 2]). However, by using the Lambert $W$ function [5], $f(x)$ is very easily inverted. The Lambert $W$ function is defined as the inverse function of $x e^{x}$ and it is implemented in MATHEMATICA by the commands ProductLog or LambertW. The Lambert $W$ function is a multivalued function that presents, for real arguments, two branches: $W_{0}(x)$ (principal branch) and $W_{-1}(x)$. Figure 2 shows the plot of both branches.

Let us consider now on a little more general function than (1), but, for simplicity, only for positive arguments; that is,

$$
f_{\beta}(x)=y=x^{-\beta x}, \quad \beta \in \mathbb{R}, x>0 .
$$

Figure 3 shows the plot of $f_{\beta}(x)$ for different values of $\beta$. It is easy to prove that

$$
\begin{aligned}
& f_{\beta}(1)=1, \\
& \lim _{x \rightarrow 0^{+}} f_{\beta}(x)=1, \\
& f_{\beta}^{\prime}(x)=0 \longleftrightarrow x=\frac{1}{e}, \quad \beta \neq 0, \\
& \lim _{x \rightarrow 0^{+}} f_{\beta}^{\prime}(x)= \begin{cases}+\infty, & \beta>0, \\
0, & \beta=0, \\
-\infty, & \beta<0,\end{cases}
\end{aligned}
$$

which agrees with Figure 3.

Solving (23), we have

$$
\begin{aligned}
-\frac{\log y}{\beta} & =x \log x \\
& =e^{\log x} \log x .
\end{aligned}
$$

Applying now the Lambert $W$ function and taking into account (26), we obtain

$$
\begin{aligned}
W\left(-\frac{\log y}{\beta}\right) & =\log x \\
& =-\frac{\log y}{\beta x},
\end{aligned}
$$

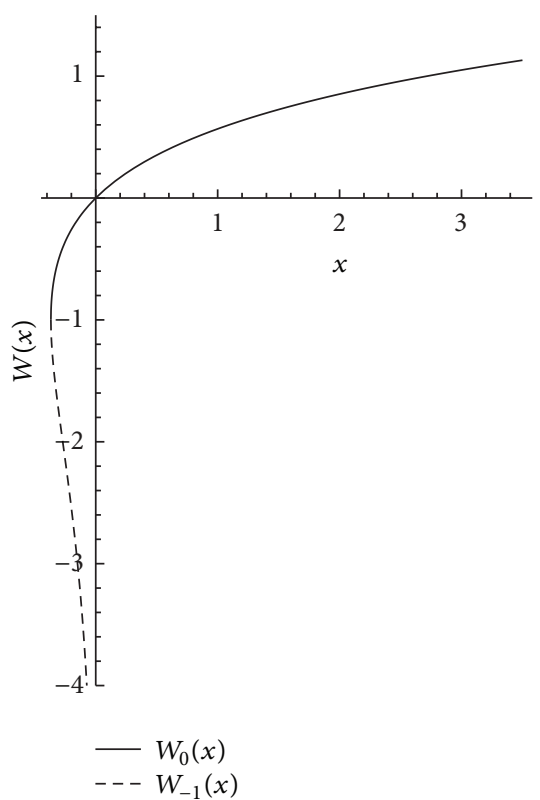

FIGURE 2: Branches of the Lambert $W$ function for real arguments.

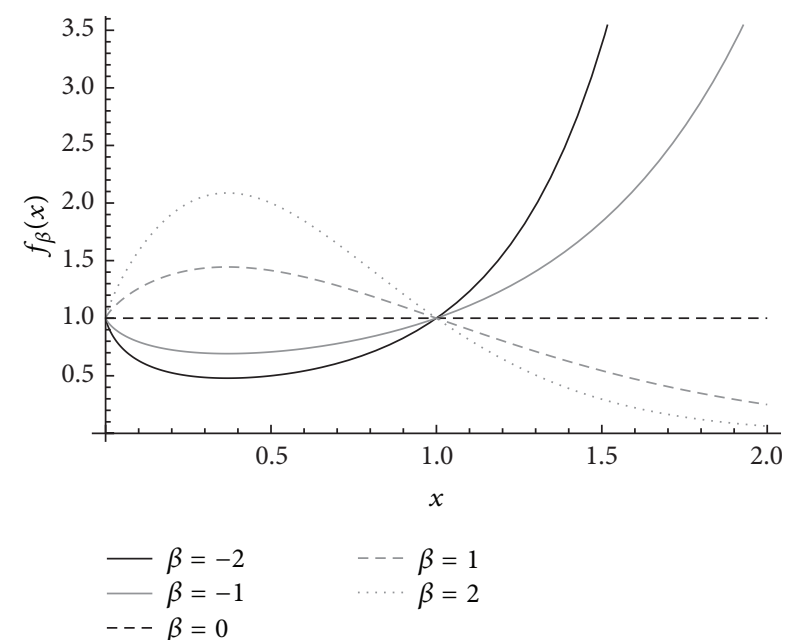

Figure 3: Plot of $x^{-\beta x}$ for different values of $\beta$.

and thus

$$
\begin{aligned}
f_{\beta}^{-1}(x) & =\exp \left(W\left(-\frac{\log x}{\beta}\right)\right) \\
& =\frac{-\log x / \beta}{W(-\log x / \beta)} .
\end{aligned}
$$

According to Figure 3, notice that, depending on the value of $x, f_{\beta}^{-1}(x)$ sometimes is a double-valued function, so we have two real values of the Lambert $W$ function in (29), that is, $W_{0}(x)$ and $W_{-1}(x)$. In this latter case, we have used the notation $W_{-1,0}(x)$. Also, from Figure 3, we can see that $f_{\beta}^{-1}(x)$ is a single-valued function for $x>1$ when $\beta<0$ and 
for $x \in(0,1)$ when $\beta>0$. Therefore, taking into account (24) and Figure 3, we can consider the following cases.

(i) Consider $\beta<0$,

$$
f_{\beta}^{-1}(x)= \begin{cases}\frac{-\log x / \beta}{W_{-1,0}(-\log x / \beta)}, & \bar{x} \leq x \leq 1, \\ \frac{-\log x / \beta}{W_{0}(-\log x / \beta)}, & x>1 .\end{cases}
$$

(ii) Consider $\beta>0$,

$$
f_{\beta}^{-1}(x)= \begin{cases}\frac{-\log x / \beta}{W_{-1,0}(-\log x / \beta)}, & 1 \leq x \leq \bar{x}, \\ \frac{-\log x / \beta}{W_{0}(-\log x / \beta)}, & 0<x<1,\end{cases}
$$

where we have defined

$$
\bar{x}=f_{\beta}\left(\frac{1}{e}\right)=e^{\beta / e} .
$$
of (1)

By setting $\beta=-1$, in (30), we obtain the inverse function

$$
f^{-1}(x)= \begin{cases}\frac{\log x}{W_{-1,0}(\log x)}, & e^{-1 / e} \leq x \leq 1, \\ \frac{\log x}{W_{0}(\log x)}, & x>1 .\end{cases}
$$

Curiously, (33) is just the closed expression given in [6] for the following power tower:

$$
g(x)=x^{(1 / x)^{(1 / x)},},
$$

which converges if and only if

$$
e^{-1 / e} \leq x \leq e^{e} .
$$

In order to see this, consider the power tower

$$
h(x)=x^{x^{x^{\prime \prime}}},
$$

which converges if and only if $[7,8]$

$$
e^{-e} \leq x \leq e^{1 / e} .
$$

Taking in (36) the logarithm of both sides and plugging back the $h(x)$ function definition, we obtain

$$
\log h=x^{x^{x^{\prime \prime}}} \log x=h \log x .
$$

Performing now the change of variables $h=e^{-u}$, we get

$$
u e^{u}=-\log x .
$$

Now, by using the principal branch of Lambert $W$ function, we can solve, for $h(x)$,

$$
h(x)=\frac{-W_{0}(-\log x)}{\log x},
$$

but, from (34) and (40), we arrive at (33):

$$
g(x)=\frac{1}{h(1 / x)}=\frac{\log x}{W_{0}(\log x)},
$$

which, according to (37), converges if and only if

$$
e^{-e} \leq \frac{1}{x} \leq e^{1 / e},
$$

that is, the interval given in (35).

In [2, Equation 02.03], $f^{-1}(x)$ is termed as coupled root function. Since the latter reference is unaware of the closed expression (33), it performs numerically the following limit [2, Equation 02.07]:

$$
\lim _{x \rightarrow \infty} \frac{f^{-1}(x)}{\log x}=0,
$$

in order to show that $f^{-1}(x)$ goes to infinity at a lower rate than logarithms. In fact, (43) is quite easily proved applying (33) and the property

$$
\lim _{x \rightarrow \infty} x e^{x}=\infty \longrightarrow \lim _{x \rightarrow \infty} W_{0}(x)=\infty,
$$

so that

$$
\lim _{x \rightarrow \infty} \frac{f^{-1}(x)}{\log x}=\lim _{x \rightarrow \infty} \frac{1}{W_{0}(\log x)}=0 .
$$

\section{Indefinite Integral}

4.1. Indefinite Integral of $f_{\beta}^{-1}$. For $\beta<0$, according to (30), let us calculate

$$
\int_{1}^{z} f_{\beta}^{-1}(x) d x=\int_{1}^{z} \frac{-\log x / \beta}{W_{0}(-\log x / \beta)} d x, \quad z>1 .
$$

Performing the change of variables $u=-\log x / \beta$, we have

$$
\int_{1}^{z} f_{\beta}^{-1}(x) d x=-\beta \int_{0}^{-\log z / \beta} \frac{u e^{-\beta u}}{W_{0}(u)} d u,
$$

and expanding the exponential function $e^{-\beta u}$, in (47), we obtain

$$
\int_{1}^{z} f_{\beta}^{-1}(x) d x=\sum_{n=0}^{\infty} \frac{(-\beta)^{n+1}}{n !} \int_{0}^{-\log z / \beta} \frac{u^{n+1}}{W_{0}(u)} d u .
$$

Performing now the change of variables $W_{0}(u)=s$ (i.e., $\left.u=s e^{s}\right)$, we get

$$
\int_{1}^{z} f_{\beta}^{-1}(x) d x=\sum_{n=0}^{\infty} \frac{(-\beta)^{n+1}}{n !} \int_{0}^{\alpha} s^{n}(s+1) e^{(n+2) s} d s,
$$

where we have set

$$
\alpha:=W_{0}\left(-\frac{\log z}{\beta}\right)
$$


The integral given in (49) can be expressed in terms of the lower incomplete gamma function [9, Equation 8.2.1]. Consider

$$
\gamma(a, z):=\int_{0}^{z} t^{a-1} e^{-t} d t, \quad \operatorname{Re} a>0
$$

thus, performing the change of variables $-t=k s$, we get

$$
\begin{aligned}
\int_{0}^{a} s^{m} e^{k s} d s & =(-k)^{-1-m} \int_{0}^{-k a} t^{m} e^{-t} d t \\
& =(-k)^{-1-m} \gamma(m+1,-k a) .
\end{aligned}
$$

Therefore, applying (52) in (49), we obtain

$$
\begin{aligned}
\int_{1}^{z} f_{\beta}^{-1} & (x) d x \\
= & \sum_{n=0}^{\infty} \frac{\beta^{n+1}}{n !(n+2)^{n+1}} \\
& \times\left\{\gamma(n+1,-(n+2) \alpha)-\frac{\gamma(n+2,-(n+2) \alpha)}{n+2}\right\} .
\end{aligned}
$$

Applying now the property [9, Equation 8.8.1]

$$
\gamma(a+1, z)=a \gamma(a, z)-z^{a} e^{-z},
$$

we may rewrite (53) as

$$
\begin{aligned}
& \int_{1}^{z} f_{\beta}^{-1}(x) d x \\
& \quad=\sum_{n=0}^{\infty} \frac{\beta^{n+1}}{n !}\left\{\frac{\gamma(n+1,-(n+2) \alpha)}{(n+2)^{n+2}}+\frac{(-\alpha)^{n+1}}{n+2} e^{(n+2) \alpha}\right\} .
\end{aligned}
$$

In order to compute the lower incomplete gamma function given in (55), we may use [1, Equation 45:4:1]

$$
\gamma(n, x)=(n-1) !\left[1-e_{n-1}(x) e^{-x}\right],
$$

where $e_{n}(x)$ is the exponential polynomial and it is given by the power-series expansion of the exponential function by truncation after the $n$th term [1, Equation 26:12:2]:

$$
e_{n}(x)=\sum_{k=0}^{n} \frac{x^{k}}{k !}
$$

Therefore, we finally obtain

$$
\begin{aligned}
\int_{1}^{z} f_{\beta}^{-1}(x) d x & \\
=\sum_{n=0}^{\infty} \frac{\beta^{n+1}}{n+2} \times\left\{\frac{1}{(n+2)^{n+1}}\right. & \\
& \left.+\left(\frac{(-\alpha)^{n+1}}{n !}-\frac{e_{n}(-(n+2) \alpha)}{(n+2)^{n+1}}\right) e^{(n+2) \alpha}\right\},
\end{aligned}
$$

where $\alpha=W_{0}(-\log z / \beta), z>1$ and $\beta<0$.
Notice as well that, for $\beta>0$, according to (31), we have to calculate

$$
\int_{z}^{1} f_{\beta}^{-1}(x) d x=\int_{z}^{1} \frac{-\log x / \beta}{W_{0}(-\log x / \beta)} d x, \quad z \in(0,1),
$$

but, according to (58), we have

$$
\begin{aligned}
& \int_{z}^{1} f_{\beta}^{-1}(x) d x \\
& =-\sum_{n=0}^{\infty} \frac{\beta^{n+1}}{n+2} \\
& \quad \times\left\{\frac{1}{(n+2)^{n+1}}+\left(\frac{(-\alpha)^{n+1}}{n !}-\frac{e_{n}(-(n+2) \alpha)}{(n+2)^{n+1}}\right) e^{(n+2) \alpha}\right\},
\end{aligned}
$$

where $\alpha=W_{0}(-\log z / \beta), z \in(0,1)$ and $\beta>0$.

4.2. Indefinite Integral of $f_{\beta}$. The indefinite integral

$$
\int x^{x} d x
$$

cannot be expressed in terms of a finite number of elementary functions [10]. Moreover, closed expression for (61) in the usual literature does not seem to be found. However, it can be expressed in closed form by using the upper incomplete gamma function [9, Equation 8.2.1]:

$$
\Gamma(a, z):=\int_{z}^{\infty} t^{a-1} e^{-t} d t, \quad \operatorname{Re} a>0 .
$$

Notice that if $z=0$ and $a=n$ is a positive integer, then we recover the usual gamma function:

$$
\Gamma(n, 0)=\Gamma(n)=(n-1) !, \quad n \in \mathbb{Z}^{+} .
$$

We can generalize (61), calculating the integral

$$
\begin{array}{r}
\int_{0}^{z} f_{\beta}(x) d x=\int_{0}^{z} \exp (-\beta x \log x) d x \\
z>0, \quad \beta \in \mathbb{R} .
\end{array}
$$

Expanding in power-series the exponential function given in (64) and integrating term by term, we get

$$
\int_{0}^{z} f_{\beta}(x) d x=\sum_{n=0}^{\infty} \frac{(-\beta)^{n}}{n !} \int_{0}^{z}(x \log x)^{n} d x .
$$

Performing now the change of variables $x^{n+1}=e^{-t}$, we obtain

$$
\begin{aligned}
& \int_{0}^{z} f_{\beta}(x) d x \\
& \quad=\sum_{n=0}^{\infty} \frac{(-\beta)^{n}(-1)^{n+1}}{(n+1) !(n+1)^{n}} \int_{\infty}^{-(n+1) \log z} e^{-t} t^{n} d t \\
& \quad=\sum_{n=1}^{\infty} \frac{\beta^{n-1}}{n ! n^{n-1}} \int_{-n \log z}^{\infty} e^{-t} t^{n-1} d t .
\end{aligned}
$$


By using now the definition of the upper incomplete gamma function (62), we arrive at

$$
\int_{0}^{z} x^{-\beta x} d x=\sum_{n=1}^{\infty} \frac{\beta^{n-1}}{(n-1) ! n^{n}} \Gamma(n,-n \log z) .
$$

In [11, Lemma 10.6], we find a similar expression for the indefinite integral of the power tower

$$
\int x^{x^{x}} d x=\sum_{n=1}^{\infty} \frac{(-1)^{n-1} n^{n-2}}{(n-1) !} \Gamma(n,-\log x) .
$$

Taking $z=1$ in (67) and using (63), we recover the expression given by [12, Equation 3.342]:

$$
\int_{0}^{1} x^{-\beta x} d x=\sum_{n=1}^{\infty} \frac{\beta^{n-1}}{n^{n}}
$$

Moreover, taking $\beta= \pm 1$, in (69), we recover the expressions given for the sophomore's dream [13, pp. 4, 44], discovered in 1697 by Johann Bernoulli, as follows:

$$
\int_{0}^{1} x^{\mp x} d x=\sum_{n=1}^{\infty} \frac{( \pm 1)^{n-1}}{n^{n}} .
$$

In order to compute the upper incomplete gamma function given in (67), we may use [1, Equation 45:4:2]:

$$
\Gamma(n, x)=(n-1) ! e^{-x} e_{n-1}(x)
$$

where $e_{n}(x)$ is the exponential polynomial (57). Therefore, (67) can be rewritten as

$$
\int_{0}^{z} x^{-\beta x} d x=\frac{1}{\beta} \sum_{n=1}^{\infty}\left(\frac{\beta z}{n}\right)^{n} e_{n-1}(-n \log z) .
$$

Let us now proceed to give another expression for the indefinite integral of $f_{\beta}$ by using the results given in (58) and (60). First, let us consider the cases $\beta<0$ and $z>1$, splitting (64) into two summands, as follows:

$$
\begin{aligned}
\int_{0}^{z} x^{-\beta x} d x & =\int_{0}^{1} x^{-\beta x} d x+\int_{1}^{z} x^{-\beta x} d x \\
& =\sum_{n=1}^{\infty} \frac{\beta^{n-1}}{n^{n}}+\int_{1}^{z} x^{-\beta x} d x
\end{aligned}
$$

where the first integral in (73) has been substituted by (69) and the second integral can be computed by knowing that $f_{\beta}(x)$ is an increasing function for $x>1$ when $\beta<0$.

Indeed, according to Figure 4, we have

$$
z \times z^{-\beta z}=1+\int_{1}^{z} f_{\beta}(x) d x+\int_{1}^{z^{-\beta z}} f_{\beta}^{-1}(x) d x
$$

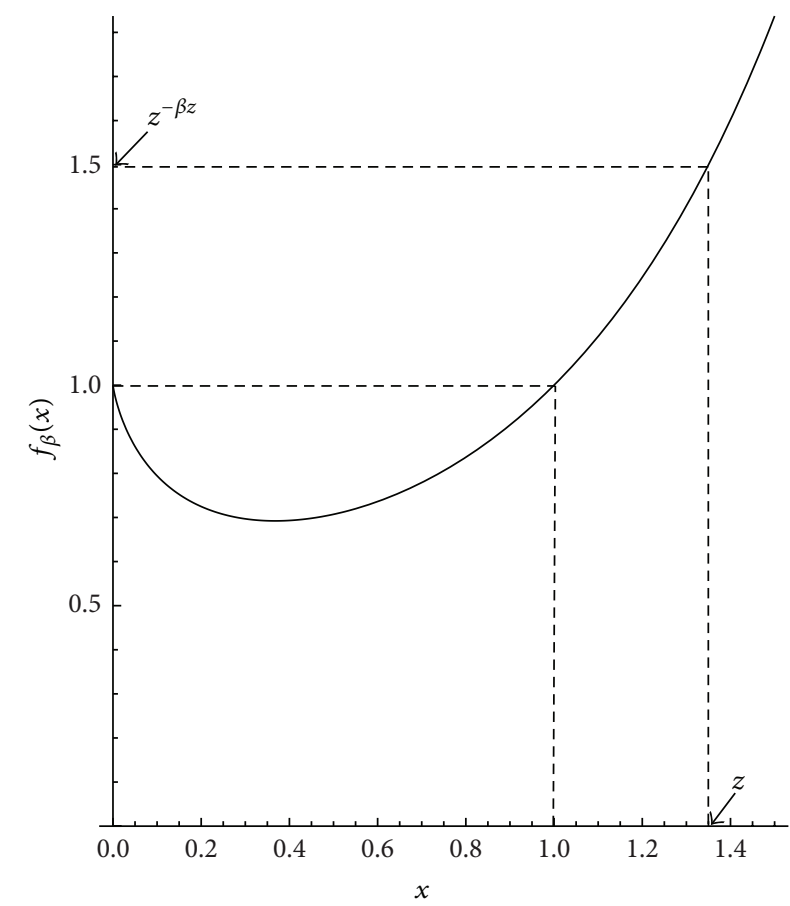

FIGURE 4: Scheme for the integration of $f_{\beta}(x), \beta<0$, beyond $z>1$.

So, from (73) and (74) and taking into account (58), we get

$$
\begin{aligned}
& \int_{0}^{z} x^{-\beta x} d x \\
& =z^{1-\beta z}-1+\sum_{n=1}^{\infty} \frac{\beta^{n-1}}{n^{n}} \\
& -\sum_{n=0}^{\infty} \frac{\beta^{n+1}}{n+2}\left\{\frac{1}{(n+2)^{n+1}}\right. \\
& \left.\quad+\left(\frac{(-\alpha)^{n+1}}{n !}-\frac{e_{n}(-(n+2) \alpha)}{(n+2)^{n+1}}\right) e^{(n+2) \alpha}\right\} .
\end{aligned}
$$

Since the following series is a telescoping series:

$$
\sum_{n=0}^{\infty}\left(\frac{\beta^{n}}{(n+1)^{n+1}}-\frac{\beta^{n+1}}{(n+2)^{n+2}}\right)=1,
$$

we can simplify (75), obtaining

$$
\begin{aligned}
& \int_{0}^{z} x^{-\beta x} d x= z^{1-\beta z} \\
&-e^{\alpha} \sum_{n=0}^{\infty} \frac{\left(\beta e^{\alpha}\right)^{n+1}}{n+2}\left\{\frac{(-\alpha)^{n+1}}{n !}\right. \\
&\left.-\frac{e_{n}(-(n+2) \alpha)}{(n+2)^{n+1}}\right\},
\end{aligned}
$$

where $\alpha=W_{0}(z \log z), z>1$ and $\beta<0$. 


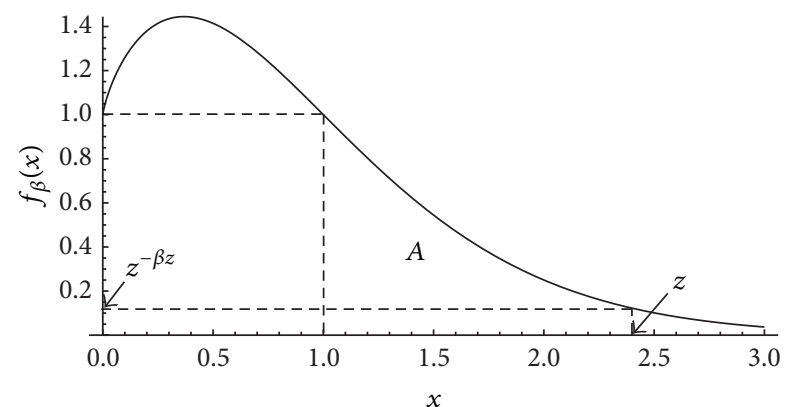

FIgURE 5: Scheme for the integration of $f_{\beta}(x), \beta>0$, for $z>1$.

For the case of $\beta>0$, according to Figure 5, we have

$$
\int_{1}^{z} x^{-\beta x} d x=\underbrace{\int_{z^{-\beta z}}^{1} f_{\beta}^{-1}(x) d x-\left(1-z^{-\beta z}\right)}_{A}+(z-1) z^{-\beta z} .
$$

Therefore, substituting (78) in (73) and taking into account (60) and (76), we arrive again at (77), but for $\beta>0$. Moreover, the range $z>1$ can be extended up to the point where $f_{\beta}$ is a monotonic increasing or decreasing function. So, according to (24), we can say that $z>1 / e$. Then, collecting all these results, we can conclude that

$$
\begin{aligned}
\int_{0}^{z} x^{-\beta x} d x= & z^{1-\beta z} \\
& -e^{\alpha} \sum_{n=0}^{\infty} \frac{\left(\beta e^{\alpha}\right)^{n+1}}{n+2}\left\{\frac{(-\alpha)^{n+1}}{n !}-\frac{e_{n}(-(n+2) \alpha)}{(n+2)^{n+1}}\right\},
\end{aligned}
$$

where $\alpha=W_{0}(z \log z), z>1 / e$, and $\beta>0$.

\section{Conflict of Interests}

The authors declare that there is no conflict of interests regarding the publication of this paper.

\section{References}

[1] K. Oldham, J. Myland, and J. Spanier, An Atlas of Functions, Springer, New York, NY, USA, 2nd edition, 2008.

[2] J. A. Fantini and G. C. Kloepfer, The Coupled Exponential, 1998, http://eretrandre.org/rb/files/JayFantini1998_203.pdf.

[3] G. H. Hardy, Orders of Infinity, Cambridge Press, England, UK, 1910.

[4] M. D. Meyerson, “The $x^{x}$ spindle," Mathematics Magazine, vol. 69, no. 3, pp. 198-206, 1996.

[5] R. M. Corless, G. H. Gonnet, D. E. Hare, D. J. Jeffrey, and D. E. Knuth, "On the Lambert $W$ function," Advances in Computational Mathematics, vol. 5, no. 4, pp. 329-359, 1996.

[6] I. N. Galidakis and E. W. Weisstein, "Power Tower," Math World-Wolfram Web Resources, http://mathworld.wolfram .com/PowerTower.html.
[7] L. Euler, "De serie Lambertina Plurimisque eius insignibus proprietatibus," Acta Academiae Scientarum Imperialis Petropolitinae, vol. 2, pp. 29-51, 1783, Reprinted in L. Euler, Opera Omnia, Series Prima, Volume 6: Commentationes Algebraicae, pp. 350369.

[8] G. Eisenstein, "Entwicklung von $\alpha^{\alpha^{\alpha "}}$, , Journal für die Reine und Angewandte Mathematik, vol. 28, pp. 49-52, 1844.

[9] F. W. J. Olver, D. W. Lozier, R. F. Boisvert, and C. W. Clark, Eds., NIST Handbook of Mathematical Functions, Cambridge University Press, New York, NY, USA, 2010.

[10] E. A. Marchisotto and G.-A. Zakeri, "An invitation to integration in finite terms," College Mathematics Journal, vol. 25, pp. 295-308, 1994.

[11] I. N. Galidakis, "On an application of Lambert's $W$ function to infinite exponentials," Complex Variables. Theory and Application, vol. 49, no. 11, pp. 759-780, 2004.

[12] I. S. Gradshteyn and I. M. Ryzhik, Table of Integrals, Series, and Products, Academic Press, New York, NY, USA, 7th edition, 2007.

[13] J. Borwein, D. Bailey, and R. Girgensohn, Experimentation in Mathematics: Computational Paths to Discovery, A K Peters, Wellesley, Mass, USA, 2004. 


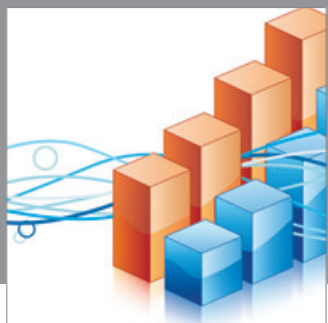

Advances in

Operations Research

mansans

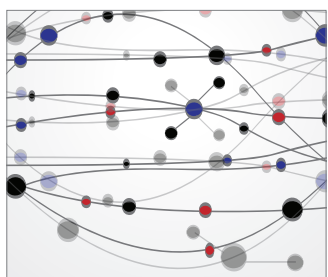

The Scientific World Journal
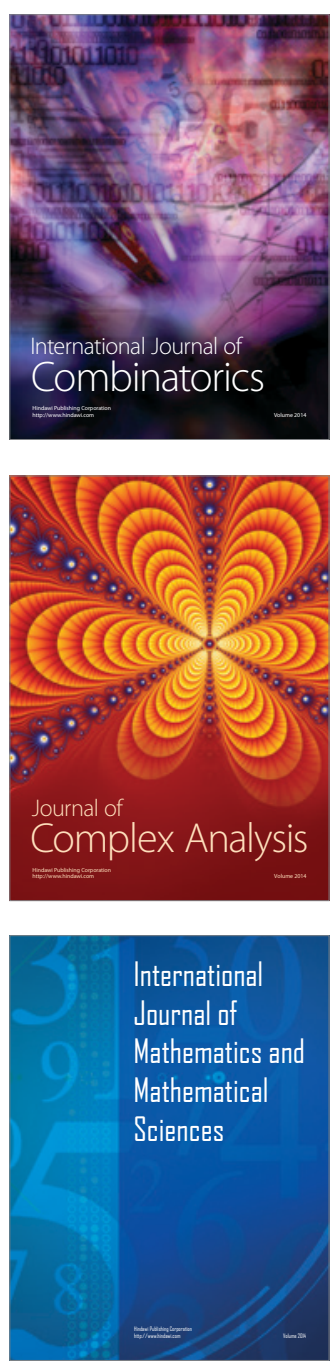
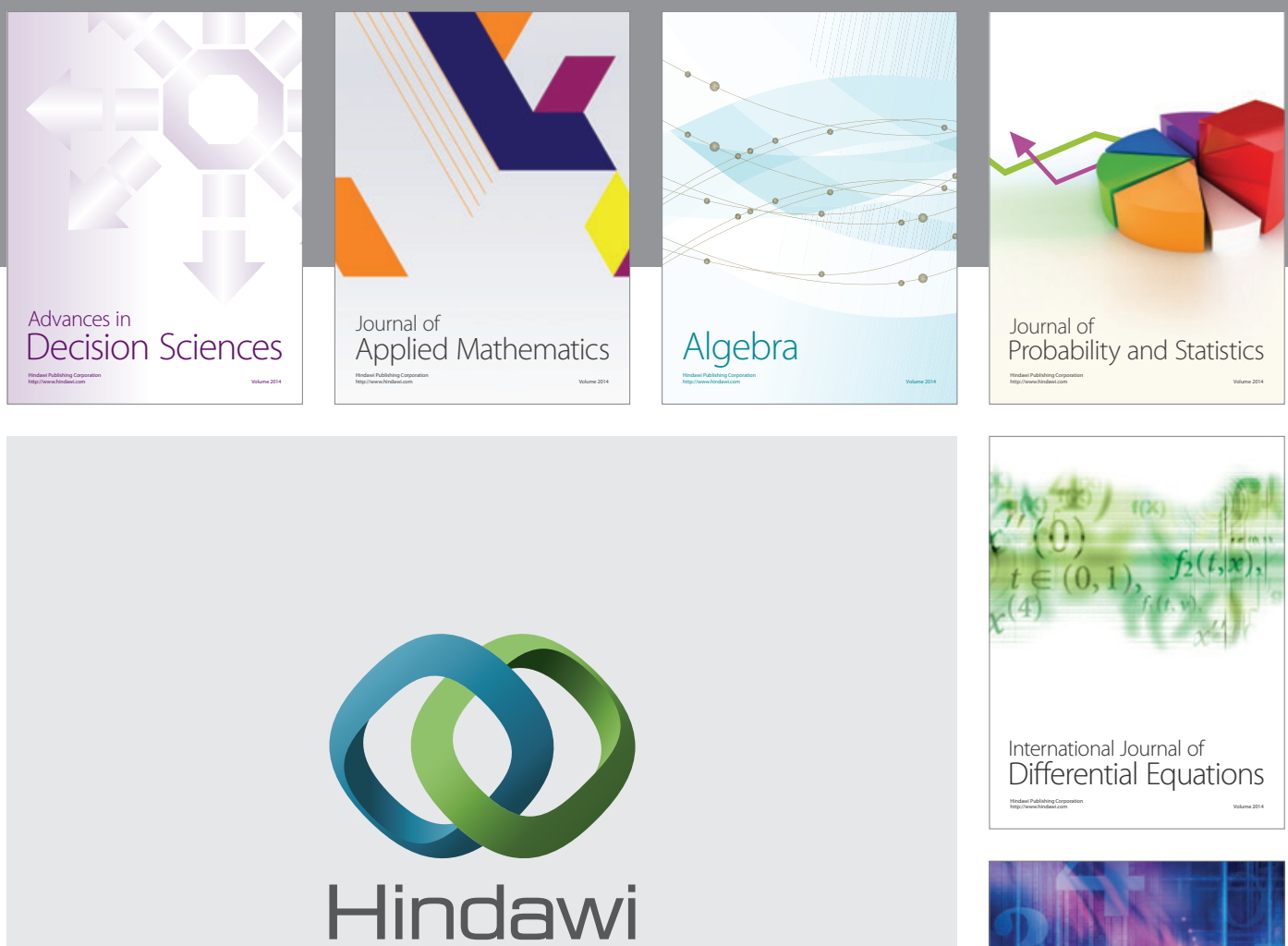

Submit your manuscripts at http://www.hindawi.com
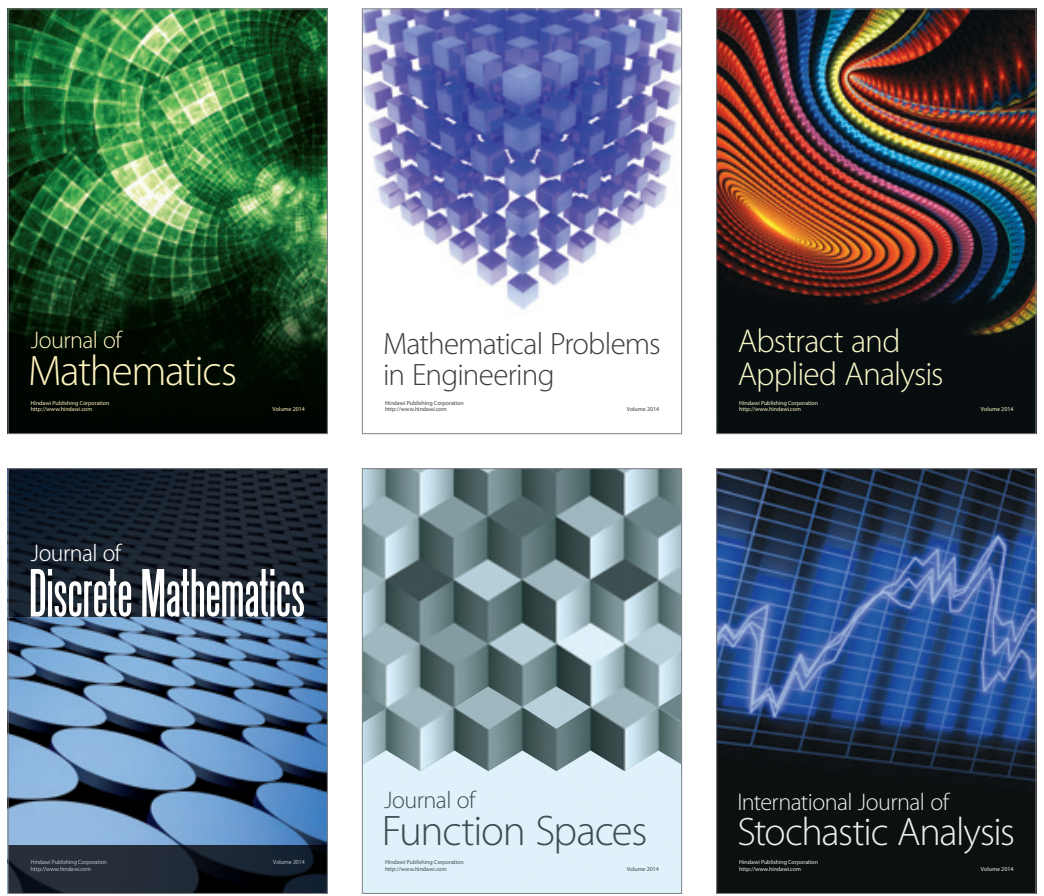

Journal of

Function Spaces

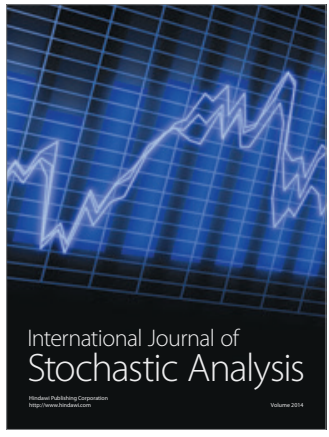

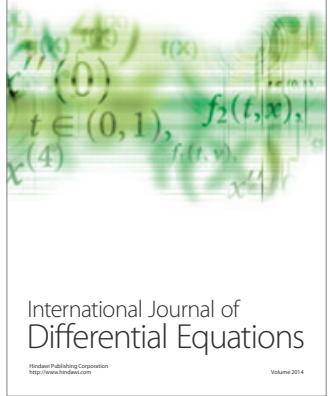
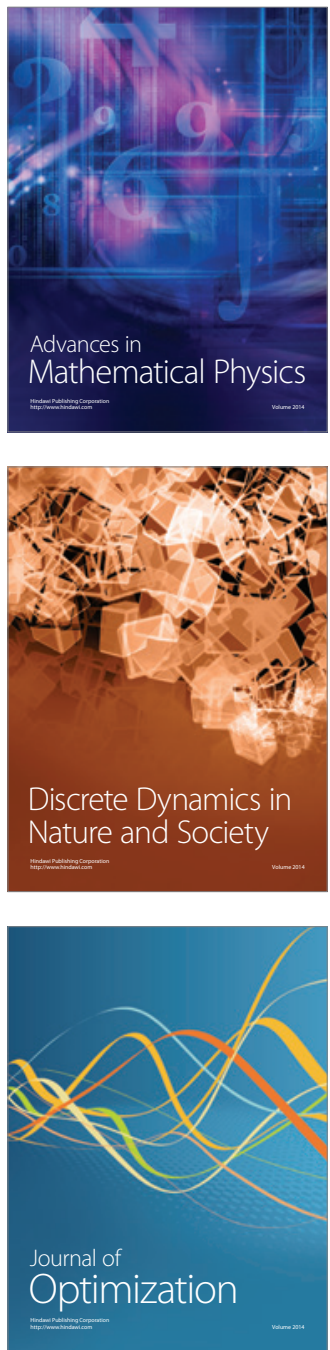